\section{Atom Chips}

C.J. Vale, B.V. Hall, D.C. Lau, M.P.A. Jones, J.A. Retter and E.A. Hinds SCOAP, University of Sussex, Brighton, United Kingdom

$T^{n}$ the last decade atomic physics has undergone a renaissance. Nobel Prizes in Physics were awarded to Cohen-Tannoudji, $\mathrm{Chu}$, and Phillips in 1997 for laser cooling and trapping of atoms and to Ketterle, Cornell, and Wieman in 2001 for Bose-Einstein condensation (BEC) in dilute alkali gases. New experimental methods unlocked through this research have resulted in an explosive growth in the field of cold and ultra-cold atoms. At $\mu \mathrm{K}$ temperatures atoms have sufficiently low energy that their de Broglie wave nature becomes evident. Such atoms can be controlled by atom optical elements (lenses, mirrors, diffraction gratings, waveguides and interferometers) in analogy with light.

One area that has been particularly exciting recently is the development of integrated atom optical devices for the storage, manipulation and controlled interaction of atoms onboard a micro-fabricated structure, the "Atom Chip"[1,2]. Miniature current-carrying wire structures or permanent magnet structures can be used to create microscopic magnetic potentials that control atoms. Experimenters are currently trying to realise beam splitters and interferometers for BEC on board atom chips. Such devices offer new levels of measurement sensitivity and have been suggested as a possible way to implement quantum information processing [3]. In this article, we give a brief review of important developments in the field atom chips and describe some of the atom chip experiments performed in our group at Sussex.

Atoms with a magnetic moment $\mu$ experience an interaction potential, $-\mu \cdot B$, in the presence of a magnetic field $B$. Those atoms with their magnetic moment aligned antiparallel to the direction of the field are known as weak field seekers because their interaction energy is lowest in areas of low field. Such atoms can be trapped or guided by a local minimum of the magnetic field, which offers a convenient means of controlling them. However, the energy scale associated with this interaction is low compared to the thermal energy of room temperature atoms, so only very cold atoms ( $\mathrm{T} \leq 1 \mathrm{mK}$ ) can be manipulated this way.

With the recent advent of laser cooling and the magneto-optical trap (MOT) it has become routine to create samples of $\approx 10^{8}$ atoms at temperatures of around $50 \mu \mathrm{K}$ or lower. This provides an invaluable source of ultra-cold atoms which can be magnetically trapped using modest fields. The first demonstration of the possibilities for atom guides were the guiding of atoms released from a MOT source. A review of this earlier work can be found in [1].

An important development for atom chips was the invention of the mirror MOT [4] which provides easy preparation and loading of cold atom clouds directly onto a surface. A mirror MOT uses the same laser and quadrupole magnetic field configuration as a standard six-beam MOT, except that two of the laser beams are formed by reflection from a mirror surface. Atoms are trapped close to the surface in the region where the incident and reflected beams overlap. The mirror is typically a gold layer on a substrate which also incorporates a pattern of current-carrying wires that can be activated to create magnetic micro-traps. A picture of our $\mathrm{BEC}$ atom chip is shown in figure 1 below. One can see the gold surface of the chip, the MOT coils oriented at 45 to the mirror and the guide wire running across the centre of the chip. Four trans-
Fig. 1: The Sussex BEC atom chip.

verse wires located just underneath the surface (not visible) provide confinement of magnetically trapped clouds along the length of the guide.

A major breakthrough for atom chips came in 2001 with the realisation of BEC on board a chip

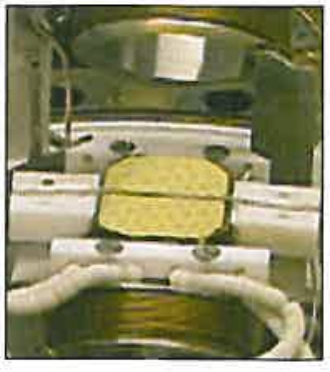
demonstrated almost simultaneously in the groups of Claus Zimmerman in Tübingen [5] and Ted Hänsch [6] in Munich. There are now several groups, including ours, who have created [2] or loaded [7] BEC on a chip and the number is growing rapidly.

In our experiments, $1 \times 10^{887} \mathrm{Rb}$ atoms are collected and cooled to $60 \mu \mathrm{K}$ in a mirror MOT $4 \mathrm{~mm}$ above the gold surface. Approximately $2 \times 10^{7}$ atoms are loaded into a magnetic micro-trap formed by the wires embedded in and under the surface. The atoms are then adiabatically compressed by ramping up the trap frequencies to $2 \pi \times 840 \mathrm{~s}^{-1}$ in the radial direction and and $2 \pi \times 26 \mathrm{~s}^{-1}$ axially. This increases the atom density and raises the elastic collision rate to $\gtrsim 30 \mathrm{~s}^{-1}$, high enough for efficient evaporative cooling to take place. We use forced rf evaporation to cool the atoms down to below $415 \mathrm{nK}$ at which point the cloud Bose condenses. The rf frequency is swept logarithmically from $13 \mathrm{MHz}$ to a final frequency of around $600 \mathrm{kHz}$ over $12.5 \mathrm{~s}$. The trap is $200 \mu \mathrm{m}$ above the surface when the condensate forms and the number of condensed atoms is typically 22000 . We view the condensate using absorption imaging, after first turning off the axial confinement and letting the atoms expand along the guide for $8 \mathrm{~ms}$. Figure 2 shows the atom cloud changing from a thermal cloud above $415 \mathrm{nK}$ to a BEC at lower temperatures.

One advantage of atom chips is that the elements producing the magnetic field are very close to the atoms. This means that the traps can have very steep potential gradients where the quantum mechanical (vibrational) modes are widely spaced, with intervals similar to the thermal energy of the atoms. For the special case of BEC, all of the atoms collect in the ground state of the trap and their behaviour is described by a single quantum mechanical wavefunction. Single mode operation is important for realising coherent quantum devices. As an example we have previously considered the case of a two-wire guide [8]. This versatile scheme consists of two wires carrying parallel currents in the presence of a transverse bias field. Simply by varying the strength of the bias field it becomes possible to split and recombine atom

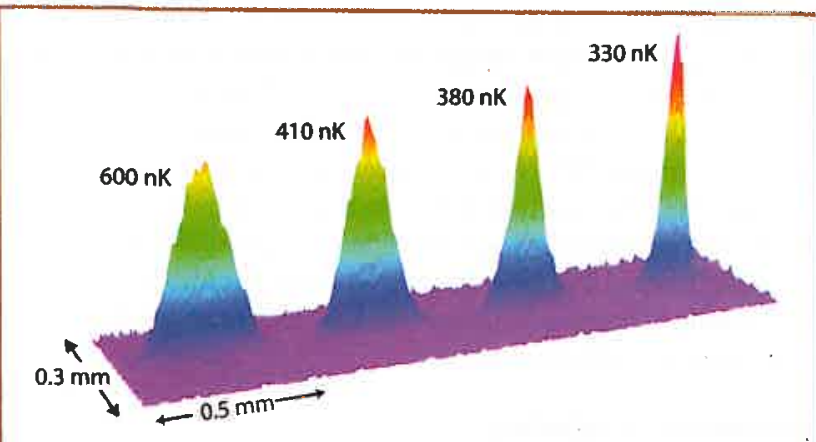

$\Delta$ Fig. 2: Density plots for four different atom clouds. Each cloud is cooled to a different temperature before being released, expanded, and imaged. The BEC starts to form at approximately $415 \mathrm{nK}$. The right-hand peak is almost entirely condensate. 
clouds, opening the possibility of making an exceedingly sensitive atom interferometer [8]. Several groups, in addition to ours, who have loaded a BEC onto an atom chip $[2,5,6,7]$ are actively pursuing quantum interferometers.

Some very recent studies in our group and others $[7,9]$ have looked at the interaction of cold atom clouds and BECs with the surface of the chip. When a very cold atom cloud or BEC is brought close to the surface, the cloud is seen to break up into fragments. These appear to be due to a small modulation of the confining potential whose origin is not yet understood. Figure 3 shows a cloud of atoms $(T=4 \mu \mathrm{K})$ released into a guide $10 \mu \mathrm{m}$ above the conductor surface. Rather than expanding freely along the guide the atoms instead gather in the potential wells that arise near the surface. We have observed atoms trapped in these surface induced wells for times greater than $200 \mathrm{~ms}$.

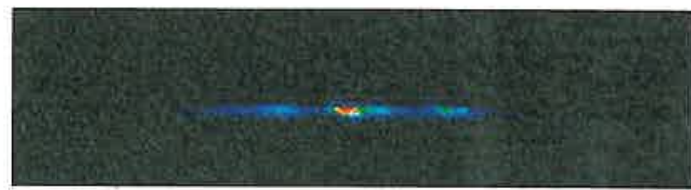

A Fig. 3: Images of a fragmented $4 \mu \mathrm{K}$ atom cloud $15 \mu \mathrm{m}$ above the guide wire.

The rapid development of atom chips based on current carrying wires is due in part to the advances made within the microcircuit fabrication industry. In our laboratory we are also pursuing atom chips where the microscopic potentials are made using commercially available magnetic storage media, most recently videotape. Permanent magnetic materials have potential advantages over current-carrying conductors as they can produce large field gradients without dissipating any energy and are without Johnson noise or temporal fluctuations. In the past we have shown that a periodically patterned magnetic surface can be used as an effective atom mirror and that such mirrors can be modu-
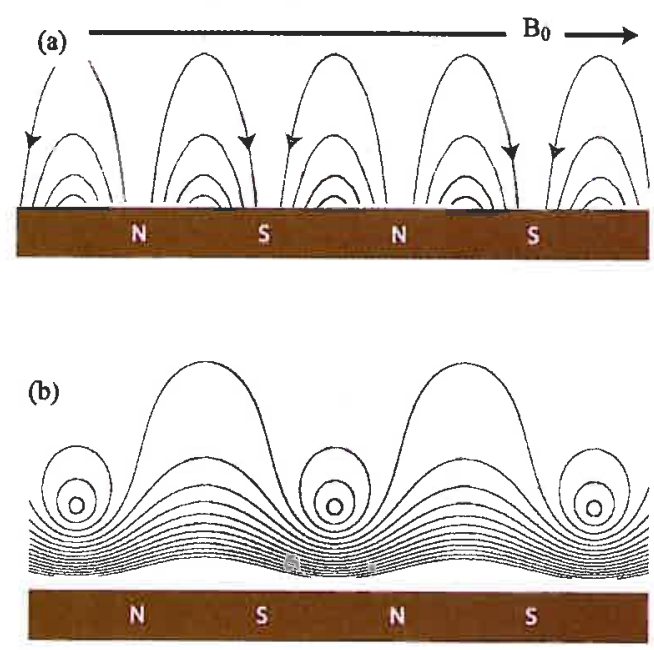

$\Delta$ Fig. 4: Fields and interaction potentials above a sinusooidally magnetised videotape. (a) A field $B_{0}$ is added to the field of the videotape itself. (b) The resulting equipotentials of the atom's interaction with the field. The circles indicate an array of tubular minima where atoms can be trapped or guided. lated through the addition of small external magnetic fields [1]. Figure 4(a) shows a uniform magnetic field $B_{0}$ added to the field of a sinusoidally magnetized videotape. At a constant height above the surface the applied field periodically cancels the field produced by the videotape. A plot of the trapping equipotentials created by this combination of fields reveals a periodic array of magnetic minima, shown in 4(b).

Atoms can be radially confined and propagated along these $2 \mathrm{D}$ magnetic guides. In our experiments two wires perpendicular to these guides make fields that confine the atoms axially. The radial gradient of the field strength in these magnetic micro-traps is $b=2 \pi B_{0} / \lambda$, where $\lambda$ is the wavelength of the magnetised recording. Even with a modest applied field, large gradients can be achieved since wavelengths as short as $10-100 \mu \mathrm{m}$ can readily be recorded (e.g. $B_{0}=1 \mathrm{mT}, \lambda=10 \mu \mathrm{m}, b=600 \mathrm{Tm}^{-1}$ ). In recent experiments with atoms in videotape micro-traps we observed three-body recombination, a loss process that requires high atom density, $10^{15} \mathrm{~cm}^{-3}$. Moreover, we observed that in the limit of low density, the lifetime of the trapped atoms was independent of distance from the magnetic surface and was independent of trap gradient. These results are in contrast to the height dependent lifetimes measured in micro-traps made by current carrying patterned wires [9].

Recording on videotape is a convenient way to achieve microscopic magnetisation patterns along one direction. However, a more versatile approach may be to use magneto-optical films. To write trapping structures onto these films a focused laser beam heats the magnetic layer, raising the temperature locally towards the Curie point. A small applied magnetic field then changes the orientation of the magnetic domains in the heated region. Scanning the laser allows an arbitrary magnetic pattern to be written, with feature sizes that can be substantially smaller than $1 \mu \mathrm{m}$. It is possible to make some parts of the surface non-magnetic by etching away the film. Atom optics based on the patterning of permanent magnetic materials is also being pursued at the Swinburne University of Technology, Australia [10].

Atom chips offer the possibility of creating new types of quantum measurement devices and many experimental groups are now working towards realising such devices. In the near future we can expect that a wide range of atom-optical elements will be integrated onto a single chip. An important next step will be to integrate optical fibres and microcavities into atom chips [11]. These will make it possible to prepare and detect individual atoms in specific quantum states so that they can form the basis of quantum logic gates.

\section{References}

[1] E.A. Hinds and I.G. Hughes, J. Phys. D: Appl. Phys. 32, R119 (1999)

[2] R. Folman et al. Adv. Atom. Mol. Opt. Phys. 48, 263 (2002)

[3] T. Calarco et al., Phys. Rev. A. 61, 022304 (2000)

[4] J. Reichel, W. Hänsel and T.W. Hänsch, Phys. Rev. Lett. 83, 3398 (1999)

[5] H. Ott et al., Phys. Rev. Lett. 87, 230401 (2001)

[6] W. Hänsel et al., Nature 413, 498 (2001)

[7] A.E. Leanhardt et al., Phys. Rev. Lett. 89, 040401 (2002)

[8] E.A. Hinds, C.J.Vale and M.G. Boshier, Phys. Rev. Lett. 86, 1426 (2001)

[9] J. Fortagh et al., arXiv:cond-mat/0205310 (2002)

[10] T.J.Davis, J.Opt.B:Quantum Semiclass.Optc. 1408 (1999)

[11] P. Horak et al., submitted to Phys. Rev. A (2002) 